\title{
Tomography of human trabecular bone with a laser-wakefield driven x-ray source
}

\author{
J. M. Cole ${ }^{1}$, J. C. Wood ${ }^{1}$, N. C. Lopes ${ }^{1,2}$, K. Poder ${ }^{1}$, \\ R. L. Abel ${ }^{3}$, S. Alatabi ${ }^{1}$, J. S. J. Bryant ${ }^{1}$, A. Jin ${ }^{4}$, \\ S. Kneip ${ }^{1}$, K. Mecseki ${ }^{1}$, S. Parker ${ }^{5}$, D. R. Symes ${ }^{6}$, \\ M. A. Sandholzer ${ }^{7}$, S. P. D. Mangles ${ }^{1}$, Z. Najmudin ${ }^{1}$ \\ 1 The John Adams Institute for Accelerator Science, Blackett Laboratory, \\ Imperial College London, London SW7 2AZ, UK \\ 2 GoLP, Instituto de Plasmas e Fusão Nuclear, Instituto Superior Técnico, \\ Universidade de Lisboa, 1049-001, Portugal \\ ${ }^{3}$ MSk Laboratory, Department of Surgery and Cancer, Charing Cross Hospital, \\ Imperial College London, London W6 8RF, UK \\ ${ }^{4}$ Department of Mechanical Engineering, City and Guilds Building, Imperial \\ College London, London SW7 2AZ, UK \\ ${ }^{5}$ Department of Physics, Imperial College London, London SW7 2AZ, UK \\ ${ }^{6}$ Central Laser Facility, Rutherford Appleton Laboratory, Didcot OX11 0QX, \\ UK \\ ${ }^{7}$ MRC Harwell, Harwell Science and Innovation Campus, Didcot OX11 0RD, \\ UK
}

\begin{abstract}
A laser-wakefield driven x-ray source is used for the radiography of human bone. The betatron motion of accelerated electrons generates x-rays which are hard (critical energy $\left.E_{c r i t}>30 \mathrm{keV}\right)$, have small source size $(<3 \mu \mathrm{m})$ and high average brightness. The x-rays are generated from a helium gas cell which is near-instantly replenishable, and thus the average photon flux is limited by the repetition rate of the driving laser rather than the breakdown of the x-ray source. A tomograph of a human bone sample was recorded with a resolution down to $50 \mu \mathrm{m}$. The photon flux was sufficiently high that a radiograph could be taken with each laser shot, and the fact that x-ray beams were produced on $97 \%$ of shots minimised failed shots and facilitated full micro-computed tomography in a reasonable time scale of several hours, limited only by the laser repetition rate. The x-ray imaging beamline length (not including the laser) is shorter than that of a synchrotron source due to the high accelerating fields and small source size. Hence this interesting laboratory-based source may one day bridge the gap between small microfocus x-ray tubes and large synchrotron facilities.
\end{abstract}

PACS numbers: 52.38.Kd, 52.38.Ph, 87.57.Q-

Keywords: Laser wakefield, betatron, x-ray, tomography, trabecular bone 


\section{Introduction}

There is a widespread need for micron scale x-ray imaging, for example in the imaging of human bone. As the population ages, degenerative diseases such as osteoporosis are becoming increasingly common and costing health services billions of pounds per year [1]. This disease is characterised by bone tissue deterioration caused by an imbalance in bone remodelling rates, leading to lowered bone density and increased fracture risk. Trabecular, or cancellous bone is a porous form of bone responsible for the transmission of forces from the joints to loadbearing surfaces. Its high surface area means it is the site of a large fraction of bone metabolic activity, and is therefore particularly sensitive to any metabolic disruption [2]. Traditional diagnosis of osteoporosis is based on the deviation from the mean of an individual's average bone density, which is an accurate predictor of fracture risk over the population. However for a particular patient up to $90 \%$ of the variation in bone strength is unexplained by average bone density [3], and it is becoming increasingly clear that the trabecular micro-architecture and distribution of mineralisation play an important role in determining individual fracture risk $[4,5]$. In addition, certain classes of drugs traditionally used in treating osteoporosis may affect patients in different ways, and it is important to understand how their bone properties are affected [6].

Detailed structural and compositional information of trabecular bone is therefore required on a perpatient basis, which cannot be accomplished with a purely 2D examination [7]. Trabecular bone is highly anisotropic, opaque, and composed of micron-scale structural elements, and therefore presents a significant imaging challenge. X-ray computed tomography $(\mathrm{CT})$ is a technique which allows the $3 \mathrm{D}$ reconstruction of the interior of a sample by recording its x-ray transmission at many angles [8]. Micro-computed tomography [9, $10](\mu \mathrm{CT})$ refers to computed tomography at high spatial resolution, the distinction commonly made for resolutions below $100 \mu \mathrm{m}$. This technique has rapidly become the dominant method for imaging the internal microstructure of bone [11]. It has been shown that it is possible to accurately characterise the 3D morphology [12], mineral distribution [13], and prevalence of micro cracks [14] within samples at high resolution. Commercial $\mu \mathrm{CT}$ scanners based on microfocus x-ray tubes operate by focussing a 30$200 \mathrm{keV}$ electron beam onto a high- $\mathrm{Z}$ anode. The anode radiates x-ray photons at a characteristic $K_{\alpha}$ energy for lower-Z materials, along with a broad bremsstrahlung component which increases in flux with Z. Scanners used for bone imaging typically use tungsten anodes where the bremsstrahlung dominates the $\mathrm{x}$-ray spectrum. The mass absorption coefficient of the sample $\mu(x, y, z)$ determines the $\mathrm{x}$-ray transmission $e^{-\int \mu \mathrm{dl}}$. Spatial variations in $\mu$ therefore generate contrast in the radiographic absorption image. The strong variation of $\mu$ with photon energy necessitates the choice of the correct $\mathrm{x}$-ray spectrum if image contrast is to be maximised.

By focussing the electron beam more strongly, the $\mathrm{x}$-ray source size is reduced and spatial resolution is increased. If the beam is too strongly focussed the anode will begin to melt, which places an upper limit on the electron beam density and therefore a lower limit on the x-ray source size. Some scanners employ rotating anodes to lower average heat loads, at the expense of larger source sizes. Others use anodes formed of liquid metal jets, which places strong restrictions on the available anode materials. Spot sizes approach $5 \mu \mathrm{m}$ in clinical microfocus sources, and up to several millimetres for larger scale full-body scanners, which at high geometric magnification is the limit on spatial resolution. This brightness limitation places a lower bound on total scan times - typically one to several hours for the type of bone sample described here.

For higher-quality imaging, researchers are increasingly performing $\mu \mathrm{CT}$ scans at synchrotron light sources $[15,16]$, where the high average source brightness is useful in several ways. More projections may be acquired in a given time, increasing scan resolution, or the same scan may be performed more quickly, increasing sample throughput. While a balance exists between scan time and resolution, at modern beamlines it is possible to achieve sub-micron resolution with a scan time of minutes. It is also possible to monochromate the $\mathrm{x}$-ray beam while maintaining a high photon flux, which readily allows quantitative tomographic measurements. Unfortunately access for routine $\mu \mathrm{CT}$ imaging is limited due to the relative scarcity and oversubscription of suitable light sources. As these synchrotron sources are driven by conventional accelerators, it is difficult to see how the size, and therefore cost, of such facilities will be reduced in the near fu- 
ture. Additionally, for applications such as bone imaging, the high brightness of a synchrotron source may be significantly more than can be used effectively, suggesting the need for an x-ray source brighter than conventional microfocus tubes but less than that delivered by a synchrotron.

In the last decade synchrotron sources based on laser-wakefield accelerators (LWFA) have witnessed rapid development. These plasma-based accelerators are able to support high electric fields which allows the acceleration of electrons to high energies over a short distance $[17,18,19,20,21]$. Furthermore they also produce synchronised ultrashort pulses of hard, bright $\mathrm{x}$-rays [22, 23]. A LWFA is formed by focussing a highintensity short-pulse laser into an underdense plasma. At sufficiently high intensities $\left(I \gtrsim 10^{19} \mathrm{Wcm}^{-2}\right)$ the pulse expels the surrounding plasma electrons entirely, creating an ion cavity, or 'bubble', travelling at the laser group velocity [24]. For a large enough bubble size, background electrons may become trapped in the bubble with some transverse momentum. The strong electromagnetic fields of the bubble cause trapped electrons to be accelerated longitudinally while oscillating transversely. These transverse 'betatron' oscillations generate synchrotron-like radiation in the forward direction, with a small divergence angle determined by the electron forward momentum and oscillation radius. The radiation spectrum on-axis has a wiggler-like shape [25] which is broad and peaks near the critical energy $E_{c r i t} \equiv \frac{3}{2} \hbar c \alpha_{\beta} k_{\beta} \gamma_{e}^{2}$, commonly chosen to characterise such spectra. Here, $k_{\beta}=$ $\omega_{p} /\left(c \sqrt{2 \gamma_{e}}\right)$ is the wavenumber associated with the betatron oscillation of electrons with Lorentz factor $\gamma_{e}, \omega_{p}$ is the plasma frequency and $\alpha_{\beta}=\gamma_{e} k_{\beta} r_{\beta}$ is the wiggler strength parameter. Accounting for the dependencies of the various parameters, $E_{\text {crit }}$ is found to scale approximately linearly with laser power and inverse plasma density [26].

The $\mathrm{x}$-ray source size is determined by the average betatron oscillation radius $r_{\beta}$, previously measured directly $[27,28]$ and indirectly $[29,30]$ to be as low as $1 \mu \mathrm{m}$. This compares favourably to commerciallyavailable microfocus tubes, which typically operate at minimum source sizes nearer $5 \mu \mathrm{m}$. The plasma formation, $\mathrm{x}$-ray generation and plasma recombination happen over a tiny fraction of a second, representing an x-ray 'anode' which is almost instantly replenishable. There is therefore no equivalent limitation on average x-ray flux as in microfocus sources.

The favourable imaging properties of betatron sources were quickly recognised, illustrated previously by high-resolution radiography of insects [31, 32], and more recently full phase-contrast tomography [33]. However the typical reported x-ray spectra of $E_{\text {crit }}<$ $10 \mathrm{keV}$ would be too soft for many medical imaging applications, including bone imaging. We have reported previously on the successful scaling of $E_{\text {crit }}$ with laser power to the point where bone imaging becomes possible [34], driven by wakefield acceleration at the near-GeV level. Here we expand upon those results and emphasise the importance of a source which is both stable and small.

\section{Beamline setup}

The experiment was performed on the Astra-Gemini laser facility at the Rutherford-Appleton Laboratory. The x-ray beamline, illustrated in figure 1, was constructed in a vacuum chamber, sealed with a $180 \mu \mathrm{m}$ thick beryllium window on the beam axis.

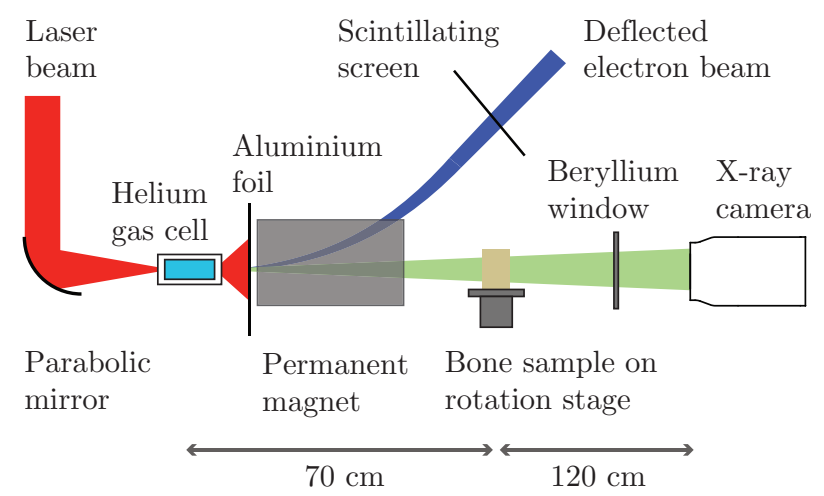

Figure 1. Experimental layout (not to scale). The laser beam (red) is focussed into a helium gas cell, producing high-energy electron (blue) and x-ray beams (green). The electrons are swept away by a $1 \mathrm{~T}$ permanent magnet, and the x-rays are deposited onto a CsI scintillator fibre-coupled to a cooled CCD.

The Gemini system delivers linearly-polarised pulses of central wavelength $800 \mathrm{~nm}$. In this experiment the typical laser energy delivered to the target was $11.4 \pm 0.4 \mathrm{~J}$ every 40 seconds (where throughout \pm denotes 1 standard deviation). The FWHM duration was $40 \pm 3 \mathrm{fs}$, and the pulse was focussed with an $f / 203 \mathrm{~m}$ focal length parabolic mirror to a spot size of $25 \times 32 \mu \mathrm{m}$ FWHM. The peak intensity at focus (in vacuum) was $(1.8 \pm 0.6) \times 10^{19} \mathrm{Wcm}^{-2}$, corresponding to a normalised vector potential $a_{0}=3.0 \pm 0.3$. The FWHM intensity contour of the focal spot contained $27 \pm 3 \%$ of the pulse energy.

A gas cell was used to confine the helium gas plasma target, the length of which was controllable remotely with a set of linear motors. The laser pulse enters and exits the cell through holes of $250 \mu \mathrm{m}$ diameter. The pressure, and therefore density, of the helium in the cell was controlled by altering the pressure of the input gas supply and measured with a fast transducer. Fine adjustments to the plasma density could be made by altering the timing of the fast gas entry valve with respect to the laser pulse. 
The plasma electron density was recorded on every shot with transverse Mach-Zehnder interferometry, and was variable from 1 to $10 \times 10^{18} \mathrm{~cm}^{-3}$. The length and density of the gas cell during the tomographic imaging was chosen for the best imaging contrast and beam reproducibility. The number of generated x-ray photons tended to increase with the gas cell length, but the image resolution was not necessarily better - possibly due to the apparent geometric increase in source size due to the longitudinal spread in x-ray emission [35]. The optimum was found near a density $n_{e}=(2.9 \pm 0.2) \times 10^{18} \mathrm{~cm}^{-3}$ for a $1.2 \mathrm{~cm}$ gas cell. We kept these parameters fixed during the tomographic scan.

Placed after the cell was a $25 \mu \mathrm{m}$ thick aluminium foil, chosen to keep x-ray absorption and electron scattering acceptably low while completely blocking the laser. This foil transmitted $73 \pm 4 \%$ of the x-ray photons above $1 \mathrm{keV}$, depending on the $\mathrm{x}$-ray spectrum. A $1 \mathrm{~T}, 15 \mathrm{~cm}$ long permanent magnet was used as part of a magnetic electron spectrometer, deflecting the electron beam away from the sample and onto a pair of scintillating Lanex screens, imaged with optical cameras.

The betatron x-ray beam freely propagated through the sample, here a $7 \mathrm{~mm}$-diameter cylindrical section of human femoral trabecular bone mounted on a rotation stage with 3 -axis translation capability. Alongside the sample were two fiducial wires, the rotation of which was used to help define the rotation axis accurately. A third wire was independently positioned near the sample, allowing for small translational corrections due to the shot-to-shot motion of the source. As a purely propagationbased imaging system image magnification was purely geometric, and chosen to be as large as possible. If parts of the sample leave the field of view the tomographic reconstruction suffers from artefacts, which places an upper limit on magnification. Xray images were taken at $1^{\circ}$ intervals from $1^{\circ}$ to $180^{\circ}$ and then at $12^{\circ}$ intervals from $180^{\circ}$ to $360^{\circ}$; the finely spaced set to enable the reconstruction, and the coarser set to more precisely determine the rotation axis. The total laser time required per scan is then approximately 2.5 hours with the Gemini laser operating at a repetition rate of $1 / 40 \mathrm{~Hz}$.

The x-ray detector was a CsI scintillator, fibrecoupled to a CCD array of $2048 \times 2048,13.6 \times 13.6 \mu \mathrm{m}$ pixels. In order to measure the x-ray spectrum a filter pack composed of 64 elemental filters was placed directly in front of the scintillator. The filters were chosen such that their $K$-edges lay within the sensitivity range of the scintillator. Assuming a synchrotron spectrum, a best-fit $E_{\text {crit }}$ was obtained by minimising the least-squares error between the expected and measured transmission for each filter [23]. Critical energies up to $E_{\text {crit }}=55 \mathrm{keV}$ were recorded over the course of the experiment. The x-ray beam profile was measured by optically imaging a LANEX screen placed along the beam axis. The source size was measured by imaging a $1 \mathrm{~mm}$-thick $\mathrm{Si}$ crystal at high magnification, and deconvolving the assumed Gaussian intensity profile from the recorded line spread function [23].

\section{Electron and x-ray beams}

The electron beams reached energies of up to $1.1 \mathrm{GeV}$ during the experiment, though during the imaging portion were typically nearer $700 \mathrm{MeV}$. Fifty consecutive shots from the tomographic imaging run are displayed in figure 2a), demonstrating the stability and consistency of the gas cell as a plasma target. Individual spectra are plotted in figure $2 \mathrm{~b}$ ), exhibiting a characteristic high-energy peak along with a broad low energy background.
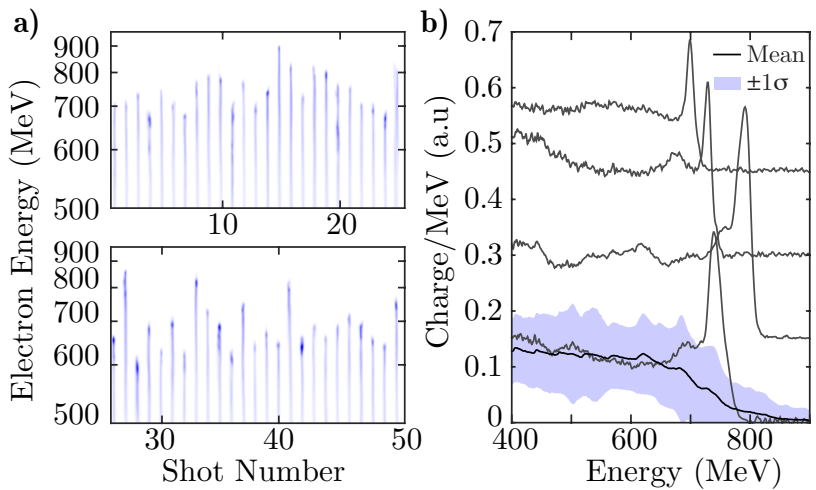

Figure 2. a) Raw electron spectra from 50 consecutive shots demonstrating the stability of the gas cell target. b) Four characteristic electron spectra offset vertically for clarity, along with the averaged spectrum.

During this run 234 laser shots were fired with a standard deviation in energy of $\pm 3.2 \%$ (as a percentage of the mean energy). Electron beams of energies greater than $400 \mathrm{MeV}$ were produced on $97 \%$ of the shots. Electron energy here is defined as the energy of the characteristic peak in the electron spectrum. The mean beam energy was $711 \mathrm{MeV}$ with a standard deviation of $9.5 \%$, and $87 \%$ of the electron beam energies were between 600 and $800 \mathrm{MeV}$.

The critical energy of the x-ray beam under the same conditions as the imaging run was measured as $33 \pm 12 \mathrm{keV}$, had divergence $10 \times 20 \mathrm{mrad}$, and exhibited pointing fluctuations of $\pm 2.1 \times 1.5 \mathrm{mrad}$ (horizontal $\times$ vertical). The total number of photons above $1 \mathrm{keV}$ was measured as $1.3 \pm 0.5 \times 10^{9}$. The FWHM x-ray source size was estimated to have an upper bound in the range of $2-3 \mu \mathrm{m}$, consistent with 
the electron oscillation amplitude required to produce the observed spectrum, as well as being in line with theoretical predictions. During the imaging run x-ray beams were produced on 227 shots, which represents $99.5 \%$ of the shots producing electron beams above $400 \mathrm{MeV}$, or $97 \%$ of the total laser shots. Without this level of consistency it would rapidly become impossible to perform a tomographic scan in a reasonable time period. The x-ray photon number and energy varied by $\pm 35 \%$, significantly higher variations than the electron beam energy, which in turn varied more than the input laser energy. The nonlinearities present in the self-injection and betatron emission processes likely act to amplify any slight differences in initial plasma conditions, emphasising the requirement that laser and plasma properties should be carefully controlled for imaging applications. Nevertheless, we demonstrate here that the level of stability so far achieved is sufficient for 3D imaging applications.

\section{Imaging results}

An example betatron image of the sample is displayed in figure 3a), alongside an image from a conventional $\mu \mathrm{CT}$ scanner (Bruker SkyScan 1172, $4.8 \mu \mathrm{m}$ voxel size, $100 \mathrm{kV}$ tube voltage, $100 \mu \mathrm{A}$ beam current) in figure $3 \mathrm{~b}$ ) and a macro optical photograph of the sample in figure 3c). At a geometric magnification of 2.7, in the betatron image each CCD pixel represents $4.8 \times 4.8 \mu \mathrm{m}$ at the sample plane. a)

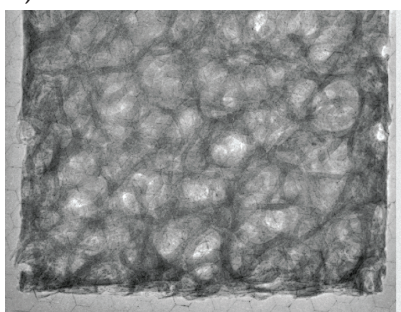

c)

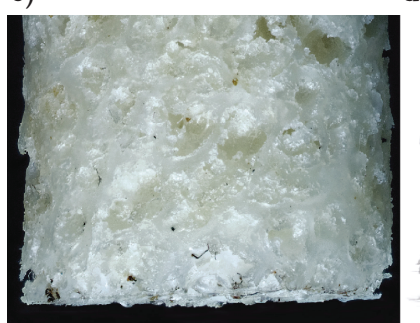

b)

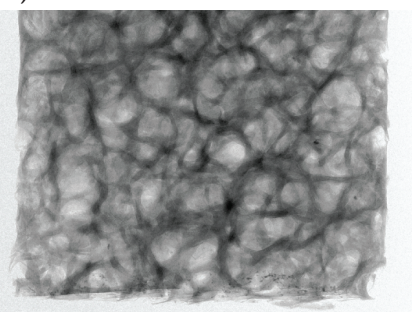

d)

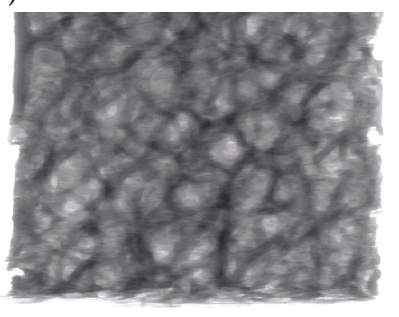

Figure 3. Images of the bone sample recorded with a) the betatron x-ray source b) conventional $\mu C T$ scanning $\mathrm{c}$ ) composite macro photography d) virtual illumination of the 3D reconstruction by a source of $E_{\text {crit }}=33 \mathrm{keV}$.

The FWHM of the line spread function of the betatron image is a measure of the spatial resolution, a) $-1 \mathrm{~mm}$

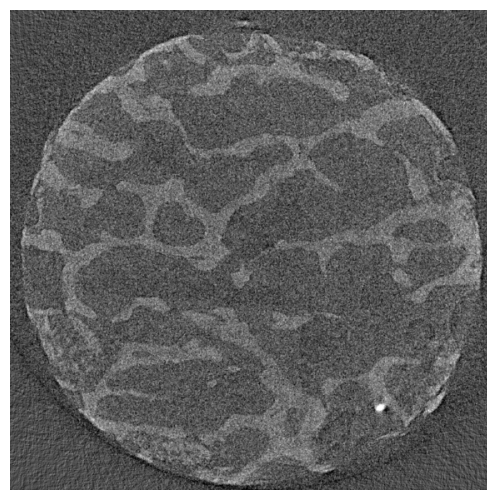

c)

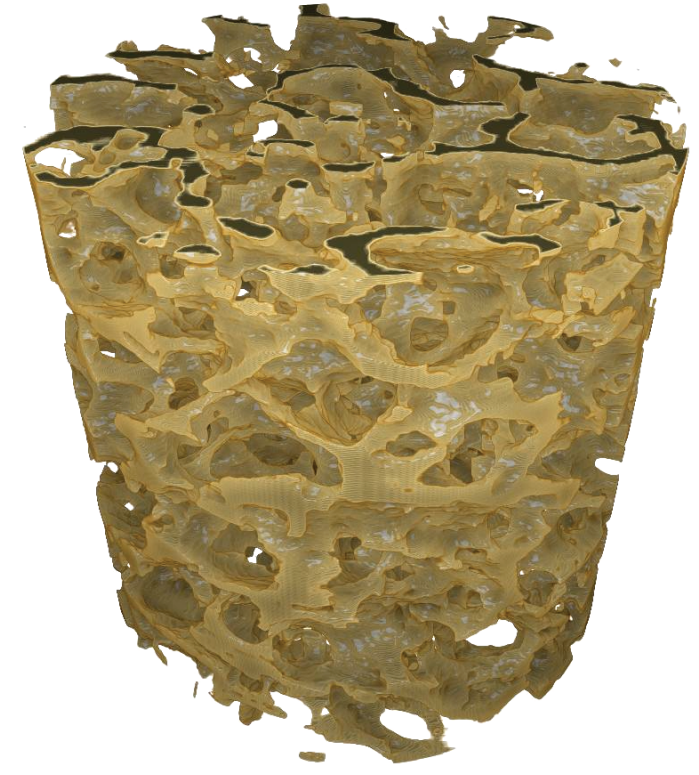

b) $-1 \mathrm{~mm}$

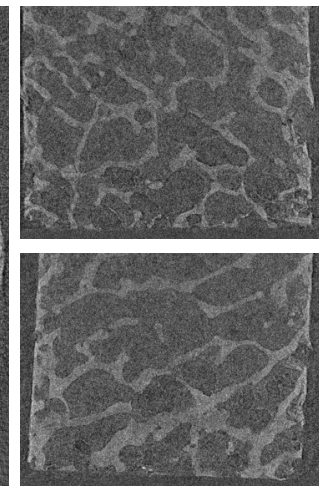

Figure 4. Visualisation of $3 \mathrm{D}$ reconstruction a) transverse slice b) coronal and sagittal slices c) full volume rendering.

and can be measured from the hard edge of the fixed fiducial wire. For the imaging run this was measured to be approximately 8 pixels, or $36 \pm 7 \mu \mathrm{m}$ at the object plane. This is close to the reported spatial resolution of the x-ray camera scintillator, implying that this is the limiting factor on resolution here.

After background subtraction and noise removal, prior to reconstruction the $\mathrm{x}$-ray projection images were individually translated to negate the small motion of the source $( \pm 4 \mu \mathrm{m})$. In this case the random source motion is useful, as it mitigates the systematic effects of cold or hot pixels on the CCD. Indeed, modern $\mu \mathrm{CT}$ scanners often incorporate random sample motion in order to lessen the impact of the reconstruction artefacts caused by faulty CCD pixels. Each image is then converted to an effective line integral of $\mu$ by dividing by the beam profile and taking the logarithm.

Each row of the image is analysed separately as a distinct slice of the bone sample, which may be denoted 
by a 2D function $f(x, y)$, and a projection of this function at angle $\theta$ as $P_{\theta}(r)$. The concatenation of all $P_{\theta}(r)$ for $\theta=1^{\circ}$ to $180^{\circ}$ is known as a sinogram, and is the input to the reconstruction algorithm. Any image defects fixed in position on the CCD appear as lines in the sinogram, and result in the appearance of spurious 'rings' in the reconstruction. Here we applied a CWTbased filter [36] to the sinogram prior to reconstruction step which effectively removes these artefacts. Based on the inverse Radon transform, in the two-step process of filtered back-projection [37], the projections are first filtered to form

$Q_{\theta}(r)=\int_{-\infty}^{\infty} \tilde{P}_{\theta}(w)|w| e^{2 \pi i w r} \mathrm{~d} w=\mathcal{H}\left(\frac{\partial P_{\theta}(r)}{\partial r}\right)$

where $\tilde{P}_{\theta}(w)$ is the Fourier transform of $P_{\theta}(r)$ and $\mathcal{H}$ denotes the Hilbert transform. The filtered projections are then 'smeared' radially and summed to reconstruct $f(x, y)$ as

$f(x, y)=\sum_{\theta} Q_{\theta}(x \cos \theta+y \sin \theta)$.

A transverse slice $f(x, y)$ of the reconstruction is plotted in figure 4a), and it is clear that despite the polychromatic nature of the x-ray source, beam hardening effects around the edges of the reconstruction are minimal. This is likely to be due to a combination of the laser-blocking foil removing the majority of the photons below $10 \mathrm{keV}$ and the falling sensitivity of the detector at high energies acting as a spectral bandpass filter. Slices in the orthogonal coronal and sagittal planes are displayed in figure $4 \mathrm{~b}$ ), demonstrating the significant anisotropy and complex internal structure of the sample. The complete tomographic dataset is visualised in figure 4c) as a volume rendering, where all transverse slices are stacked into a $3 \mathrm{D}$ voxel array.

It is difficult to quantitatively assess the quality of the reconstruction given the complex internal structure of the sample, but it is possible to create a synthetic projection image from the reconstruction, taking into account the sample absorption and camera sensitivity which can then be directly compared to the experimental images and the sample itself. When displayed alongside one another in figure 3a)-d), it is clear that the reconstruction represents an accurate model of the sample. Examination of the line spread functions of sharp features in the reconstruction suggests a maximum 3D resolution of approximately $50 \mu \mathrm{m}$. Resolution is worsened slightly at the edge of the sample, where the finite divergence of the beam causes the projection to be averaged transversely over approximately 15 pixels, or $70 \mu \mathrm{m}$ at the sample. This can be ameliorated in future with the use of 'cone beam' reconstruction methods [38]. At larger geometric magnifications the spatial coherence of the source will cause some diffraction around hard edges in the image. In that case the images should be back-propagated before reconstruction. Diffuse x-ray scattering by the sample was not taken into account.

\section{Discussion}

One of the main advances made in this experiment when compared to previous work is the successful scaling of $E_{\text {crit }}$ to a medically relevant level by using a higher-power laser system. The importance of this fact is underlined by the form of the signal-to-noise ratio (SNR) of the tomographically-reconstructed bone density [39]

$S N R \propto \sqrt{\frac{N \mu^{2}}{L e^{\mu L}}}$

where $N$ is the number of photons incident on the sample, $\mu$ is the mass absorption coefficient of the sample, here assumed spatially constant, and $L$ is the sample thickness. Maximising the SNR minimises the required dose for a given measurement precision, and therefore is an important quantity to optimise when considering in-vivo imaging. Maximising the SNR for a given photon flux requires an optimal $\mu \propto L^{-1}$. Over the medically relevant energy range, the mass absorption coefficient of a sample typically varies as $\mu(E) \propto E^{-3}$, so finding a $\mu$ which maximises the SNR is equivalent to using photons of an optimal energy. The transmission curve of this sample is plotted in figure 5a), alongside the SNR scaling predicted by 3 in figure $5 \mathrm{~b}$ ). Photons with energies below $\approx$ $20 \mathrm{keV}$ are almost completely absorbed, and so do not contribute towards the generation of absorption contrast. Similarly photons above $\approx 80 \mathrm{keV}$ are almost completely transmitted and contribute a nearconstant background. In the intermediate energy range there is a strong peak in SNR near $30 \mathrm{keV}$, and it is in this energy range that image contrast is maximised and dose levels are minimised. Samples of different thicknesses and compositions have different optimal imaging energies, though the optimum is less pronounced for thicker samples. Nevertheless, the potential to vary the photon energy is a further attraction of this source.

The second factor to consider in optimising SNR is the total number of photons $N$, equal to the product of the number of photons per projection image and the total number of projections recorded. The simplest path to improved reconstruction quality then is acquiring as many projections as possible, though of course this comes at the expense of increased dose and scan time. As the source here is pulsed the exposure time for each projection is effectively zero, and so the limiting factor on the total scan time is the repetition 


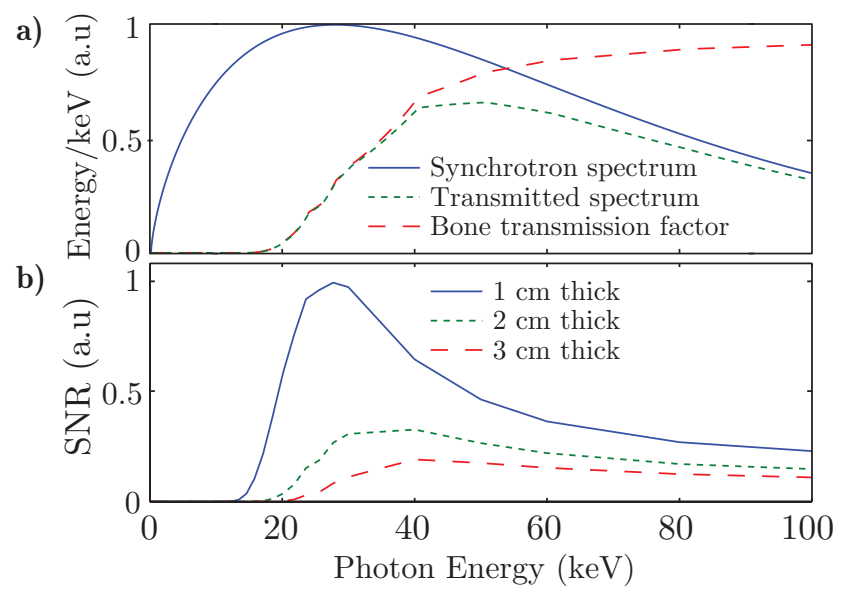

Figure 5. a) The average transmission function of the bone sample as applied to a synchrotron spectrum with $E_{\text {crit }}=$ $33 \mathrm{keV}$. b) The signal-to-noise ratios (SNR) of bone samples of varying thickness according to 3 , normalised to the peak SNR of a $1 \mathrm{~cm}$ thick sample.

rate of the laser. A relevant figure of merit is the average brightness of the source, defined as the number of photons delivered per second per $\operatorname{mrad}^{2}$ of solid angle per $\mathrm{mm}^{2}$ of source area. For the laser driven source, integrating over the medically-relevant energy range of $10-100 \mathrm{keV}$, comprising $64 \%$ of the photons above $1 \mathrm{keV}$ at $E_{\text {crit }}=33 \mathrm{keV}$, we measure an average brightness of $11_{-6}^{+12} \times 10^{10} \mathrm{~s}^{-1} \mathrm{~mm}^{-2} \mathrm{mrad}^{-2}$ and an average flux of $5.9 \pm 2.4 \times 10^{5} \mathrm{~s}^{-1} \mathrm{mrad}^{-2}$. In figure 6 the average brightness of the laser source is plotted in the brightness-source size plane, both for the current experiment and for a variety of microfocus sources, adapted from $[40,41]$. The solid line represents the simulated upper limit of a tungsten anode microfocus tube, using measured anode melting points [42] and simulated spectra [43]. It is clear that a laser system can already deliver competitive x-ray brightnesses at a small source size. However if Gemini were to operate at a $10 \mathrm{~Hz}$ repetition rate as has been proposed [44], it would become several orders of magnitude brighter than other laboratory-scale sources. Though of course, this comes at the expense of new challenges, including higher gas loading on the vacuum system and potential cumulative laser damage to the gas cell.

Though not discussed in detail here, the high spatial coherence of betatron x-ray beams naturally lend themselves to phase-contrast x-ray imaging due to their consequent increased spatial coherence. Phase contrast tomography of mixed objects including bone and soft tissue is challenging due to the disparity in attenuation between tissues, though more advanced reconstruction techniques have been shown to be effective for both synchrotron $\mu \mathrm{CT}$ and conventional $\mu \mathrm{CT}$ augmented with x-ray diffraction gratings [45]. This is an imaging regime suitable for exploration with

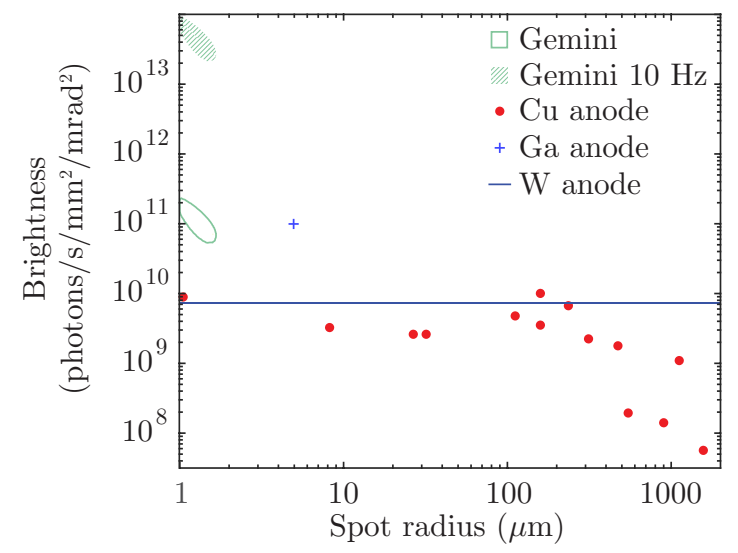

Figure 6. Brightness of various sources as a function of source size. The measured average brightness of the betatron source is indicated by the green ringed region, and that of an upgraded Gemini by the hatched region. The points are experimental data from low-Z anode microfocus sources, and the line taken from a simulation of a high-Z tungsten anode source.

betatron sources, and one we plan to investigate in the future.

Finally, it should be possible to increase the resolution of the reconstruction in the near future. In this experiment the source size was smaller than a voxel and so does not contribute to the measured resolution, which is instead dominated by the Poisson photon noise and scintillator PSF. Increasing the resolution to the CCD limit would be possible by acquiring more projection images. The ultimate resolution limit depends on the imaging geometry and detector consider the layout sketched in figure 7 where a source of size $s$ is backlighting an object $d_{1}$ away, forming an image on a detector at a further distance $d_{2}$. The geometric magnification $M=h_{2} / h_{1}=\left(d_{1}+d_{2}\right) / d_{1}$, and a point in the object casts an effective 'shadow' of size $r=s d_{2} / d_{1}$ on the detector. Taking into account the geometric magnification, the source-size-limited resolution is then $r / M=s d_{2} /\left(d_{1}+d_{2}\right)$. If the detector has pixel size $p$, the effective minimum resolution determined by the detector is $p / M=p d_{1} /\left(d_{1}+d_{2}\right)$. The overall image resolution is the larger of these two quantities for a given imaging geometry, and reaches a minimum of $s p /(s+p)$ when $d_{1}=s d_{2} / p$. At this point the total beamline length is $d_{1}+d_{2}=d_{2}(1+s / p)$. The sample has some finite thickness, implying a minimum for $d_{2}$, and in this case the total beamline length for highest resolution scales with the source size.

In a synchrotron the source size is large, necessitating a long beamline of 10s-100s of metres. The small betatron source size means that the imaging distances need not be as large as those in a synchrotron beamline for equivalent resolution. Detector resolution is commonly boosted by using a system where a thin scintillator is imaged by a high magnification optical 


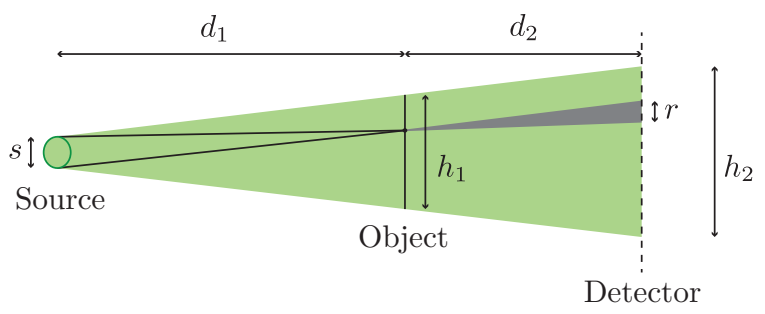

Figure 7. Layout of a generic back-illumination system. The imaging distances $d_{1}$ and $d_{2}$ in lensless x-ray imaging determine the geometric magnification and source size-limited resolution, as discussed in the text.

camera. Detector efficiency drops in this case, so it is important that the source also has high brightness if high resolution is to be achieved.

\section{Conclusion}

We have reported on the development of an x-ray source based on the betatron oscillation of laserwakefield accelerated electrons. The stability of this source was sufficient to allow us to perform a high resolution 3D tomographic scan. The source is shown to possess the right combination of spectrum, source size and brightness that highresolution micro-computed tomography of a trabecular bone sample is achieved, a significant imaging challenge for which there is pressing medical need. The anticipated development of LWFA systems operating at high repetition rates would, when applied to this imaging technique, support the construction of unique laboratory-scale high brightness x-ray sources of relevance to a wide variety of imaging applications.

\section{Acknowledgements}

This work was supported by STFC grant ST/J002062/1. R. A. was supported by the EPSRC and Wellcome Trust through the Medical Engineering Solutions in Osteoarthritis Centre of Excellence. We acknowledge the help of the staff of the CLF, Rutherford-Appleton Laboratory for assistance during the experiment.

\section{References}

[1] Blume S W and Curtis J R 2011 Osteoporosis Int. 22 $1835-44$

[2] Snyder B D and Piazza S 1993 Calcified Tissue Int. 53 $14-22$

[3] Ciarelli M J, Goldstein S A, Kuhn J L, Cody D D and Brown M B 1991 J. Orthopaed. Res. 9 674-82

[4] Kleerekoper M, Villanueva A R, Stanciu J, Rao D S and Parfitt A M 1985 Calcified Tissue Int. 37 594-97

[5] Carbonare L D and Giannini S 2004 J Endocrinol. Invest. 27 99-105
[6] Borah B, Ritman E L, Dufresne T E, Jorgensen S M, Liu S, Sacha J, Phipps R J and Turner R T 2005 Bone 37 $1-9$

[7] Odgaard A and Gundersen H J 1993 Bone 14 173-82

[8] Hounsfield G N 1973 Brit. J. Radiol. 46 1016-22

[9] Elliott J C and Dover S D 1982 J. Microsc. 126 211-13

[10] Flannery B P, Deckman H W, Roberge W G and D'Amico K L 1987 Science 237 1439-44

[11] Ritman E L 2011 Annu. Rev. Biomed. Eng. 13 531-52

[12] Chappard C, Peyrin F, Bonassie A, Lemineur G, BrunetImbault B, Lespessailles E and Benhamou C-L 2006 Osteoarth. Cartilage 14 215-23

[13] Nuzzo S, Peyrin F, Cloetens P, Baruchel J and Boivin, G 2002 Med. Phys. 292672

[14] Thurner P J, Wyss P, Voide R, Stauber M, Stampanoni M, Sennhauser U and Müller, R 2006 Bone 39 289-99

[15] Peyrin F, Salome M, Cloetens P, Laval-Jeantet A M, Ritman E L and Rüegsegger P 1998 Technol. Health Care 6 391-401

[16] Salome M, Peyrin F, Cloetens P, Odet C, Laval-Jeantet A M, Baruchel J and Spanne P 1999 Med. Phys. 262194

[17] Tajima T and Dawson J M 1979 Phys. Rev. Lett. 43 26770

[18] Mangles S P D et al 2004 Nature 431 535-38

[19] Faure J, Glinec Y, Pukhov A, Kiselev S, Gordienko S, Lefebvre E, Rousseau J-P, Burgy F and Malka V 2004 Nature 431 541-44

[20] Geddes CGR, Toth C S, Van Tilborg J, Esarey E, Schroeder C B, Bruhwiler D, Nieter C, Cary J and Leemans WP 2004 Nature 431 538-41

[21] Esarey E, Schroeder C and Leemans W P 2009 Rev. Mod. Phys. 81 1229-85

[22] Rousse A et al 2004 Phys. Rev. Lett. 93135005

[23] Kneip S et al 2010 Nature Phys. 6 980-83

[24] Pukhov A and Meyer-ter Vehn J 2002 Appl. Phys. BLasers O. 74 355-61

[25] Esarey E, Shadwick B A, Catravas P and Leemans W P 2002 Phys. Rev. E 65056505

[26] Thomas A G R 2010 Phys. Plasmas 17056708

[27] Schnell M et al 2012 Phys. Rev. Lett. 108075001

[28] Kneip S et al 2012 Phys. Rev. ST Accel. Beams 15 2-6

[29] Ta Phuoc K, Corde S, Shah R, Albert F, Fitour R, Rousseau J-P, Mercier B and Rousse A Phys. Rev. Lett. 97225002

[30] Albert F, Shah R, TaPhuoc K, Fitour R, Burgy F, Rousseau J-P, Tafzi A, Douillet D, Lefrou T and Rousse A Phys. Rev. E $\mathbf{7 7} 056402$

[31] Kneip S et al 2011 Appl. Phys. Lett. 99093701

[32] Fourmaux S et al 2011 Opt. Lett. 36 2426-28

[33] , Wenz J, Schleede S, Khrennikov K, Bech M, Thibault P, Heigoldt M, Pfeiffer F and Karsch S 2015 Nat. Commun. 67568

[34] Cole J M et al 2015 Sci. Rep. 513244

[35] Corde S et al 2012 Plasma Phys. Contr. F. 54124023

[36] Münch B, Trtik P, Marone F and Stampanoni M 2009 Opt. Express $\mathbf{1 7} 8567-91$

[37] Kak A C and Slaney M Principles of Computerized Tomographic Imaging 1988 (IEEE Press)

[38] Feldkamp L A, Davis L C and Kress J W 1984 J. Opt. Soc. Am. A 1 612-19

[39] Flynn M J, Hames S M, Reimann D A and Wilderman. J 1994 Nucl. Instrum. Meth. A 353 312-15

[40] Verman B, Jiang L and Kim B 2002 Rigaku J. 19 4-13

[41] Tuohimma T 2008 Liquid-Jet-Target Microfocus X-Ray Sources: Electron Guns, Optics and Phase-Contrast Imaging ( $\mathrm{PhD}$ Thesis, Royal Institute of Technology, Sweden)

[42] Grider D E, Wright A and Ausburn P K 1986 J. Phys. D Appl. Phys. 19 2281-92

[43] Poludniowski G, Landry G, DeBlois F, Evans P M and 
Verhaegen, F 2009 Phys. Med. Biol. 54 N433-38

[44] Banerjee S, Ertel K, Mason P D, Phillips P J, Siebold M,

Loeser M, Hernandez-Gomez C and Collier J L 2012 Opt. Lett. 372715

[45] Hahn D, Thibault P, Fehringer A, Bech M, Koehler T, Pfeiffer F and Noël P B 2015 Sci. Rep. 510452 